\title{
Fluoroscopic Analysis of Tibial Translation in Anterior Cruciate Ligament Injured Knees With and Without Bracing During Forward Lunge
}

\author{
Maryam Jalali,, ${ }^{1,{ }^{*}}$ Farzam Farahmand, ${ }^{3,4}$ Seyed Mohammad Ebrahim Mousavi, ${ }^{1}$ Seyed Ali \\ Golestanha, Tahmineh Rezaeian, ${ }^{5}$ Shahram Shirvani Broujeni, ${ }^{6}$ Mehdi Rahgozar, and \\ Fateme Esfandiarpour ${ }^{8}$
}

${ }^{1}$ Department of Orthotics and Prosthetics, University of Social Welfare and Rehabilitation Sciences, Tehran, Iran

${ }^{2}$ Iran Helal Institute of Applied Sciences and Technology, Tehran, Iran

${ }^{3}$ University of Leeds, Leeds, UK

${ }_{5}^{4}$ Research Center of Biomedical Technologies and Robotics (RCBTR), Tehran University of Medical Sciences, Tehran, Iran

${ }^{5}$ Department of Radiology, Mostafa Khomeini Hospital, Shahed University, Tehran, Iran

6 Sports Medicine Federation, Tehran, Iran

7 Department of Biostatistics and Computer, University of Social Welfare and Rehabilitation Sciences, Tehran, Iran

${ }^{8}$ Department of Physical Therapy, Ahvaz Jundishapur University of Medical Sciences, Ahvaz, Iran

${ }^{*}$ Corresponding author: Maryam Jalali, Department of Orthotics and Prosthetics, University of Social Welfare and Rehabilitation Sciences, Tehran, Iran. Tel: +98-9125040255, E-mail:marjalali@gmail.com

Received: January 25, 2014; Revised: February 27, 2014; Accepted: March 15, 2014

\begin{abstract}
Background: Despite several studies with different methods, the effect of functional knee braces on knee joint kinematics is not clear. Direct visualization of joint components through medical imaging modalities may provide the clinicians with more useful information. Objectives: In this study, for the first time in the literature, video fluoroscopy was used to investigate the effect of knee bracing on the sagittal plane kinematics of anterior cruciate ligament (ACL) injured patients.

Patients and Methods: For twelve male unilateral ACL deficient subjects, the anterior tibial translation was measured during lunge exercise in non-braced and braced conditions. Fluoroscopic images were acquired from the subjects using a digital fluoroscopy system with a rate of $10 \mathrm{fps}$. The image of each frame was scaled using a calibration coin and analyzed in AutoCAD environment. The angle between the two lines, tangent to the posterior cortexes of the femoral and tibial shafts was measured as the flexion angle. For the fluoroscopic images associated with $0^{\circ}, 15^{\circ}, 30^{\circ}, 45^{\circ}$ and $60^{\circ}$ knee flexion angles, the relative anterior-posterior configuration of the tibiofemoral joint was assessed by measuring the position of landmarks on the tibia and femur.

Results: Results indicated that the overall anterior translations of the tibia during the eccentric (down) and concentric (up) phases of lunge exercise were $10.4 \pm 1.7 \mathrm{~mm}$ and $9.0 \pm 2.2 \mathrm{~mm}$ for non-braced, and $10.1 \pm 3.4 \mathrm{~mm}$ and $7.4 \pm 2.5 \mathrm{~mm}$, for braced conditions, respectively. The difference of the tibial anterior-posterior translation behaviors of the braced and non-braced knees was not statistically significant. Conclusion: Fluoroscopic imaging provides an effective tool to measure the dynamic behavior of the knee joint in the sagittal plane and within the limitations of this study, the pure mechanical stabilizing effect of functional knee bracing is not sufficient to control the anterior tibial translation of the ACL deficient patients during lunge exercise.
\end{abstract}

Keywords: Kinematics; Brace; Anterior Cruciate Ligament; Fluoroscopy; Knee Joint

\section{Background}

Anterior cruciate ligament (ACL) is renowned as a key stabilizer of the knee joint. A large prevalence rate of 80000 to 250000 per year, or even more, has been reported for ACL injury (1), which makes this ligament the most frequently traumatized structure of the knee (2). ACL injuries are often due to sport accidents (3) and can affect the normal life of injured population who are mostly young and active persons (4), including early retirement of sports persons (2).

The effect of ACL injury on the biomechanics of the knee joint has been extensively studied in the literature. It has been shown to alter the joint's normal kinematics (5-9), functional performance, proprioception (10), and stabil- ity (11). An important consequence of the joint's biomechanics alteration is the abnormal loading which has been suggested to be responsible, at least in part, for the degenerative changes in the articular cartilage and the progressive development of knee osteoarthritis $(12,13)$. Although reconstruction surgery has been reported to be cost effective (14), there is no adequate evidence that it reduces the overall rate of osteoarthritis in long-term (14-16). However, whether a surgical or conservative approach is employed, a rehabilitation program is an inevitable part of the treatment plan and wearing functional knee braces (FKBs) has been suggested as part of this program since 70 s (17). These braces are often used for par- 
tially torn ACL patients and those who have undergone ACL graft reconstruction, immediately after surgery, to support the healing ACL, improve the joint's functional stability, and restore the normal kinematics.

The efficacy of FKBs has been assessed in previous studies using clinical and arthrometric evaluation (18-21), cadaveric examination $(22,23)$, and motion analysis using skin markers (24-27). The methodologies of these studies, however, might be reasonably criticized for being subjective, unrealistic and suffering from limited accuracy (28, 29). In a recent study, intracortical pins, inserted into the bones have been used to obtain the knee kinematics in braced and non-braced conditions (30). This methodology, although accurate, is invasive and might interfere and affect the normal behavior of the subjects.

As such, the most reliable methodology to investigate the kinematical efficacy of FKBs is to use medical imaging modalities that provide direct visualization of the position and hence the relative motion of the bones noninvasively. Imaging techniques have been employed for kinematics analysis of the ACL deficient knees in two (31) and three $(9,32-34)$ dimensions often during quasi-static activities. Also, video fluoroscopy has been recently used to measure the kinematic behavior of the normal and ACL injured knees during dynamic activities $(8,35)$.

\section{Objectives}

In this study, for the first time in the literature, video fluoroscopy is used to investigate the in-vivo kinematical behavior of ACL deficient knees, before and after wearing FKBs, in order to examine the hypothesis that FKBs reduce the anterior tibial translation of ACL deficient patents.

\section{Patients and Methods}

Twelve male volunteers with unilateral ACL injury, selected by convenient sampling among patients referred to a medical imaging center participated in this study. The number of samples was determined by priori sample-size power analysis ( $\beta=0.20$, and $\alpha=0.5$ ) based on the results of a previous study that measured the effect of wearing FKB on the anterior tibial translation of 15 normal subjects (20). All subjects were diagnosed with complete isolated ACL tear via MRI and clinical examination performed by an expert orthopedic surgeon. The exclusion criteria were age above 45 and below 18, any sign of inflammation, joint effusion, injury in meniscus, collateral or posterior cruciate ligament, concomitant injuries in the lower limb, any abnormal limb characteristics, and pain during the lunge exercise. Prior to testing, all participants read and signed an informed consent form approved by the Research Ethics Committee of the University of Social Welfare and Rehabilitation Sciences.

A functional knee brace was fabricated for each participant by a professional orthotist. The brace consisted of an anterior shank shell, a posterior thigh shell (thermoformed plastic sheets), two fastening Velcro straps on each shell, and a pair of knee bars. The shank and thigh shells were made based on the tracings of the entire limb with precise measurements of the limb contours for each subject. The orthotic knee joint was a standard polycentric hyper-extension controlling joint (17K48, Otto Bock, Germany) with bars made from non-metallic materials. The metallic screws of the orthotic knee joint were also replaced by plastic ones to prevent masking of the fluoroscopic images.

The fluoroscopic images were acquired using a digital fluoroscopy system (Baccara, DMS-Apelem, France) with a detector image array of $1024 \times 1024$ pixels and 12 bit depth, capable of imaging at 10 frames per second. Imaging was performed by the trained staff of the radiology department of Mostafa Khomeini Hospital under the supervision of a specialized interventional radiologist (MD). Subjects wore a lead apron and thyroid shield all throughout the test session. During the test, the fluoroscopy table was positioned vertically and the subject stood on a $45 \mathrm{~cm}$ height table, to position the knee joint as close to the intensifier as possible in a proper field of view $(32 \mathrm{~cm}$ diameter). The subject was then asked to perform a slow lunge exercise with his injured leg positioned forward, stay stationary with the injured knee at maximum bent for a short time, and then go back to the initial standing position. The subject was asked to keep his trunk upright throughout the maneuver. Imaging started after making sure that the knee joint was in the proper position that was seen all through the test duration. A metal coin with a known radius securely attached to the subject's leg or thigh was used to calibrate the image of each frame. Two tests were performed by each subject in non-braced and braced conditions. After application of the knee brace, the subject was allowed to walk for a couple of minutes to get used to the brace, followed by a 3-5 minutes rest before performing the experiment.

The image of each frame was exported to the AutoCAD environment (ver 2013, Autodesk Inc., Montreal, Canada) for analysis (Figure 1). The angle between the two lines, tangent to the posterior cortexes of the femoral and tibial shafts, was measured as the flexion angle. The relative anterior-posterior configuration of the tibiofemoral joint was assessed by measuring the relative position of a fixed point on the femur with respect to a local coordinate system attached to the tibia. The femoral reference point ( $C$ in Figure 1) was considered to be the center of a circle fitted to the posterior edge of the femoral intercondylar notch (36). The tibial coordination system was defined with the y axis, along the long axis of the tibial shaft, i.e. the line tangent to the posterior cortex of the tibia. The line from the most anterior point of the tibial plateau (P in Figure 1) perpendicular to the y axis was defined as the $x$ axis, and the intersection point of the $y$ and $\mathrm{x}$ axes ( $\mathrm{O}$ in Figure 1 ) as the origin of the tibial local coordination system. The difference between the x coordinates of the midpoint of OP, considered as the tibial reference point, and $\mathrm{C}$ in the tibial coordination system was used 
to indicate the relative anterior-posterior position of the tibia with respect to the femur $(36,37)$. For each subject, the fluoroscopic images associated with $0^{\circ}, 15^{\circ}, 30^{\circ}, 45^{\circ}$ and $60^{\circ}$ knee flexion angles were analyzed in two braced and non-braced conditions. For each frame, the radius of the calibration coin was used to determine the scale factor that was applied to measurement results.

The normality of the variables' distributions was tested by 1 -sample K-S test. Paired t-tests $(\alpha=0.05)$ were used to compare the mean of anterior tibial translation in braced and non-braced conditions in different knee angles ( 5 levels: $0^{\circ}, 15^{\circ}, 30^{\circ}, 45^{\circ}, 60^{\circ}$ ) and phases of the lunge maneuver [eccentric (downward) phase and concentric (upward) phase]. The time duration of the maneuver and the peak knee flexion angle were also compared by paired t tests ( $\alpha$ $=0.05)$. The reliability of the process was tested using intraclass correlation coefficient $\left(\mathrm{ICC}_{2,2}\right)$. Ten sample images were randomly chosen from the available data and two raters measured the anterior tibial translations using the methodology described above. The measurement process was repeated after a 2-week interval by each rater and the reliability was calculated by taking the average of the two measurements. All the statistical tests were performed using SPSS 17 for Windows (SPSS Inc., Chicago, IL, USA).

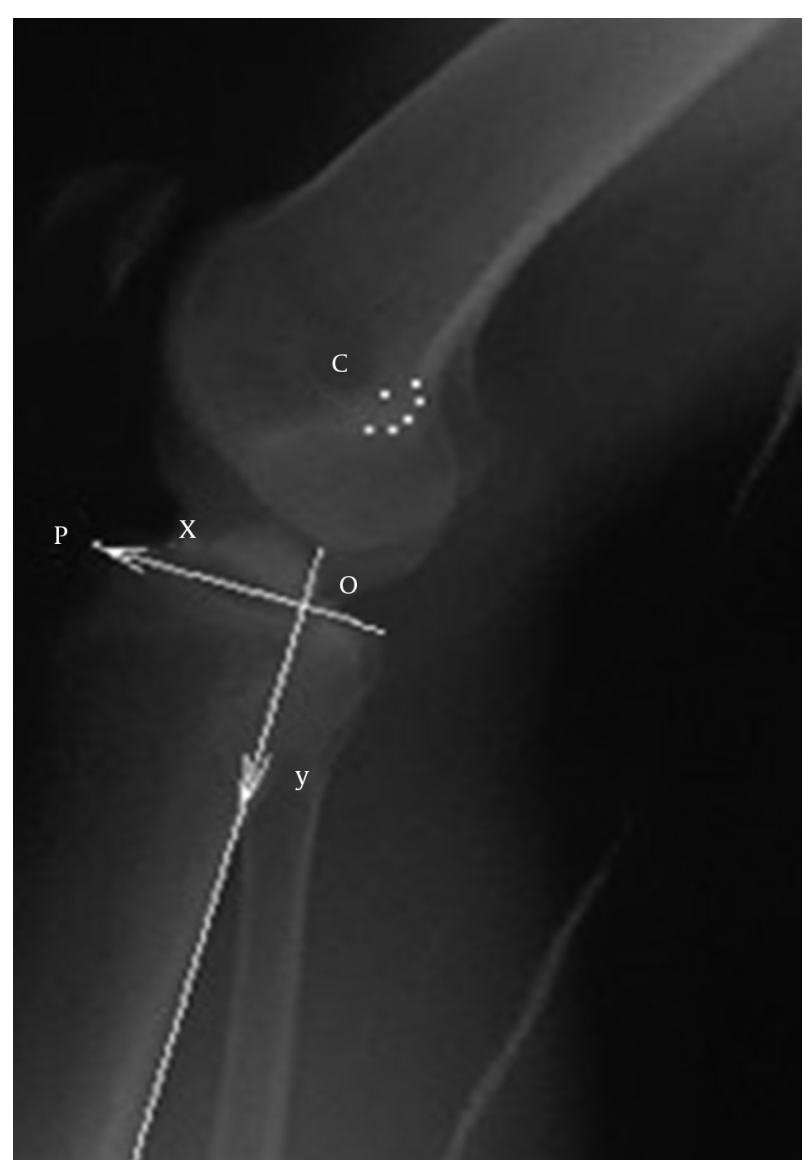

Figure 1. Landmarks on the tibia and femur that were used to measure the tibiofemoral joint configuration.

\section{Results}

The subjects were between 19 and 44 years of age with the detailed data indicated in Table 1 . All subjects completed the maneuver with no discomfort in both braced and non-braced conditions. The general characteristics of the maneuver in braced and non-braced conditions were similar. The mean time duration of the maneuver was $12.8 \pm 3.4$ seconds without brace in comparison with $12.9 \pm 3.9$ seconds when using brace $(\mathrm{P}=0.75)$. The result was the same for means of the maximum knee flexion angles with $64.6 \pm 11.6$ and $67.3 \pm 11.9$ degrees in non-braced and braced conditions, respectively $(\mathrm{P}=0.07)$.

The results obtained for the tibial anterior-posterior translations are illustrated in Figures 2 and 3. In general, an increasing tibial anterior translation was observed with progressive knee flexion in the eccentric (downward) phase of lunge maneuver, in both the non-braced and braced conditions. The overall anterior translation of the tibia during the eccentric phase was $10.4 \pm 1.7 \mathrm{~mm}$ for non-braced and $10.1 \pm 3.4 \mathrm{~mm}$ for braced knees, with no significant difference. Also, no significant difference was observed between the tibial anterior-posterior translation behaviors of the braced and non-braced knees at the flexion angles examined, except for $60^{\circ}$ at which the anterior tibial translation (ATT) of the braced knee was larger than that of the non-braced $(P=0.033)$. A similar trend was observed for tibial posterior translation during the concentric (upward) phase of the lunge maneuver, when going back from bent knee to the initial standing position. The overall posterior translation of the tibia was 9.0 $\pm 2.2 \mathrm{~mm}$ for non-braced and $7.4 \pm 2.5 \mathrm{~mm}$ for braced knees. No significant difference was observed between the tibial anterior-posterior translation behaviors of the braced and non-braced knees at any flexion angles examined. The ICC $(2,2)$ value (average measure) was $0.869(\mathrm{P}<0.001)$.

\begin{tabular}{lc}
\hline \multicolumn{2}{l}{ Table 1. Detailed Data of the Participants of the Study ${ }^{\mathrm{a}}$} \\
\hline \multicolumn{1}{l}{ Variable } & Value \\
\hline Age, $\mathbf{y}$ & $29.07 \pm 7.58$ \\
Height, cm & $176.90 \pm 7.89$ \\
Weight, kg & $74.9 \pm 7.84$ \\
\hline Injured limb & 6 \\
\hline $\mathrm{R}$ & 6 \\
\hline $\mathrm{L}$ & \\
\hline Injury type & 8 \\
\hline NC & 4 \\
\hline C & $11 \pm 6$ \\
\hline Interval from injury, month
\end{tabular}

\footnotetext{
a Abbreviations: C, contact; L, left; NC, non-contact; R, right; L, left
} 


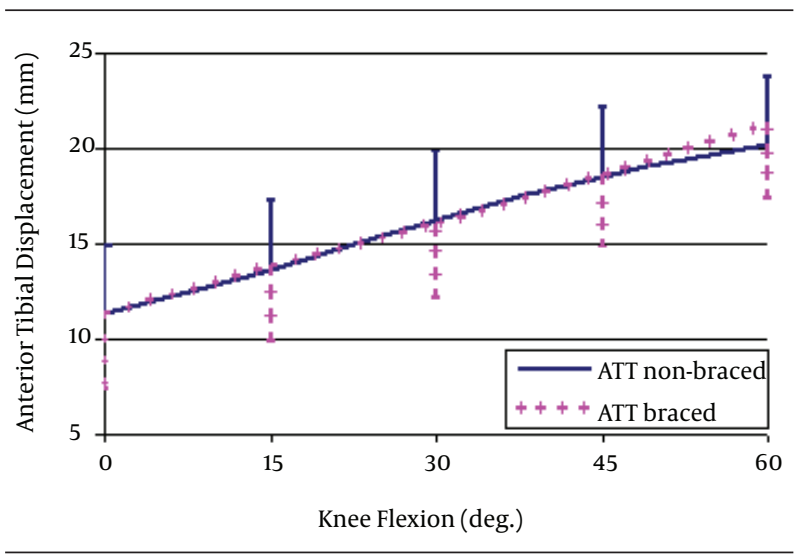

Figure 2. Anterior tibial translation in the eccentric (downward) phase of lunge.

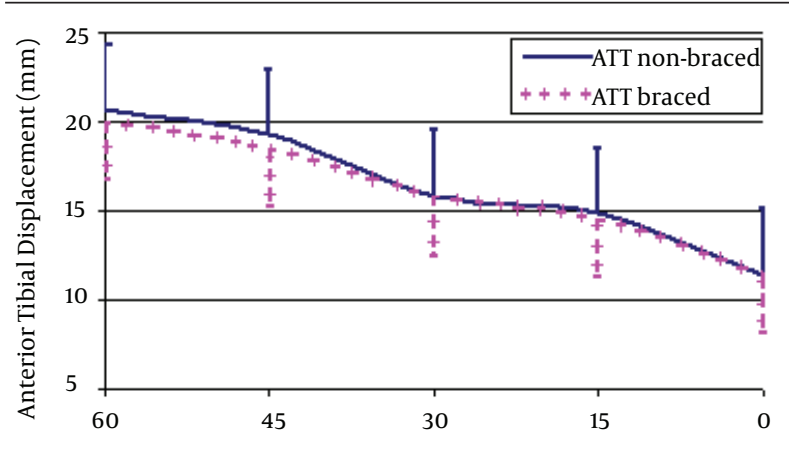

Knee Flexion (deg.)

Figure 3. Anterior tibial translation in the concentric (upward) phase of lunge.

\section{Discussion}

In this study, for the first time, fluoroscopic imaging was used to measure the effect of functional knee bracing on the tibiofemoral joint arthrokinematics to avoid uncertainties involved in the previous in-vivo investigations, e.g. skin marker artifacts and intracortical pin interferences with soft tissues. However, in spite of being non-invasive and providing direct visualization of the positions of the bones, there are concerns over limitations of this method that might give rise to inaccuracies. First of all, a single fluoroscope only provides kinematics information in two dimensions, i.e. only the movements of bones in the plane of radiography are visualized. For the ACL injured knees, one of the most important components of the joint movement is the anterior-posterior translation of the tibia relative to the femur that can be well observed in sagittal plane images. However, considering the fact that the knee joint movement is three dimensional in nature, there is a possibility that other components of movement, namely the internal-external rotations of the bones affect the sagittal plane measurements. In spite of the studies that have used single plane imaging techniques for measurement of the sagittal plane motion data of the knees (31, 35-37), the effect of such cross talk has not been studied before and we are not aware of the inaccuracies that it might have caused in our results. However, this effect is expected to be of lower importance in our comparative study of non-braced and braced knees. Further studies using accurate three dimensional measurement techniques, e.g. orthogonal fluoroscopy (6) are needed to evaluate this assumption.

Another difficulty in studying the human joint kinematics using fluoroscopy arises from technical limitations of this imaging modality. First of all, the resolution of the fluoroscopic images is limited. This can affect the accuracy of the bony landmark identification and the resultant spatial measurements. Considering the $32 \mathrm{~cm}$ diameter field of view and the $1024 \times 1024$ pixels resolution of our fluoroscopic images, the size of each pixel was about $0.3 \times$ $0.3 \mathrm{~mm}$. Thus the highest accuracy that could be expected for a linear measurement, e.g. anterior tibial translation, is $0.3 \mathrm{~mm}$. Moreover, the fluoroscopic images are blurred, not only due to the spatial blurring factors that plague all x-ray imaging devices, but also due to the persistence of video signals between TV frames, a phenomenon known as image lag (38). The latter factor can lead to motion blurred images for moving objects, especially when the speed of motion is high in comparison with the system's imaging frequency. Considering the $10 \mathrm{fps}$ frequency of our fluoroscope system, we asked the subjects to perform the lunge maneuver slowly to obtain improved results. However, our images were still affected by some blurring effects that made the accurate identification of bony landmarks difficult.

In spite of the above limitations, the results of the intraclass correlation coefficient tests indicate a high reliability for the measurement procedure employed. In addition, our results for the anterior-posterior translation behavior of the ACL injured knees are consistent with the literature. We found increasing tibial anterior translation with progressive knee flexion in both eccentric and concentric phases of the lunge maneuver (Figures 2 and 3). A similar trend has been well documented in the literature for ACL deficient knees $(6,35,36)$. Also, the extent of the tibial anterior translation found in our study for ACL injured knees is within the range of the reports in previous publications. The overall anterior translation of the tibia in the non-braced condition were $10.4 \pm 1.7 \mathrm{~mm}$ and $9.0 \pm 2.2 \mathrm{~mm}$, for eccentric and concentric knee flexion between full extension and 60 degrees, respectively, which is comparable with the 11.4 $\mathrm{mm}$ displacement reported by Vergis et al. (36) during step accenting (closed chain). Defrate et al. (6) reported a total of $14 \mathrm{~mm}$ tibial anterior translation when the knee was flexed from full extension to 90 degrees flexion in a lunge movement (closed chain).

The results of our study regarding the effect of using a FKB on the kinematics of the ACL injured knees indicated no significant difference. This result is consistent with 
the cadaveric studies of Wojtys et al. (23) that reported no significant difference in tibial translation in braced and non-braced conditions. However, they reported a significant change in tibial internal-external rotation behavior, which was not examined in our study. Besides, in the invivo study of Ramsey et al. (30), using intracortical pins, the changes in the kinematics of ACL deficient knees were minor following wearing braces with no consistent decrease in the anterior tibial translation.

Such insufficiency might seem contradicting with the clinical results suggesting improved stability and performance following wearing FKBs $(18,19)$. However, it is a fact that our results are limited to the type and speed of the functional task we examined. The brace might be more effective in high-speed and/or open chain tasks, particularly those involved with rotational instability. Also, no control group was examined in our study. It only tested the affected limbs of the subjects with and without a knee brace after a very short adaptation time. The performance of FKBs in restoring the normal kinematics of the tibiofemoral joint might improve over time after the patients get used to the brace proprioceptive feedback and learn to change their muscular contraction pattern towards a safer and closer to normal kinematics. With the above considerations in mind, much care must be taken in the generalization of our results. The authors suggest further investigations on large sample sizes of normal and ACL injured subjects, and after longer adaptation times with knee braces to examine the efficacy of the functional knee braces in more detail.

\section{Acknowledgements}

This research was conducted as part of the first authors Ph.D. program at the University of Social Welfare and Rehabilitation Sciences. The authors wish to thank Reza Vahab Kashani (orthotists), Amir Hosein Eskandari and Mohammad Akbar (software developers), Ali Shakouri Rad (Homaye Salamat Imaging Center), and the staff of the radiology department of Mostafa Khomeini Hospital and Sports Medicine Federation for providing help and facilities.

\section{Author's Contributions}

Study concept and design: Maryam Jalali, Farzam Farahmand and Fateme Esfandiarpour; acquisition of data: Maryam Jalali and Tahmineh Rezaeian; analysis and interpretation of data: Maryam Jalali and Tahmineh Rezaeian; drafting of the manuscript: Maryam Jalali; critical revision of the manuscript for important intellectual content: Farzam Farahmand, Mehdi Rahgozar, and Fateme Esfandiarpour; statistical analysis: Maryam Jalali and Mehdi Rahgozar; administrative, technical, and material support: Maryam Jalali, Farzam Farahmand, Seyed Mohammad Ebrahim Mousavi, Seyed Ali Golestanha, and Shahram Shirvani Broujeni; study supervision: Farzam Farahmand and Maryam Jalali.

\section{References}

1. Griffin LY, Albohm MJ, Arendt EA, Bahr R, Beynnon BD, Demaio $\mathrm{M}$, et al. Understanding and preventing noncontact anterior cruciate ligament injuries: a review of the Hunt Valley II meeting, January 2005. Am J Sports Med. 2006;34(9):1512-32.

2. Lam MH, Fong DT, Yung P, Ho EP, Chan WY, Chan KM. Knee stability assessment on anterior cruciate ligament injury: Clinical and biomechanical approaches. Sports Med Arthrosc Rehabil Ther Technol. 2009;1(1):20.

3. Gianotti SM, Marshall SW, Hume PA, Bunt L. Incidence of anterior cruciate ligament injury and other knee ligament injuries: a national population-based study.J Sci Med Sport. 2009;12(6):622-7.

4. Louboutin H, Debarge R, Richou J, Selmi TA, Donell ST, Neyret P, et al. Osteoarthritis in patients with anterior cruciate ligament rupture: a review of risk factors. Knee. 2009;16(4):239-44.

5. Dennis DA, Mahfouz MR, Komistek RD, Hoff W. In vivo determination of normal and anterior cruciate ligament-deficient knee kinematics. JBiomech. 2005;38(2):241-53.

6. Defrate LE, Papannagari R, Gill TJ, Moses JM, Pathare NP, Li G. The 6 degrees of freedom kinematics of the knee after anterior cruciate ligament deficiency: an in vivo imaging analysis. Am J Sports Med. 2006;34(8):1240-6.

7. Zhang LQ, Shiavi RG, Limbird TJ, Minorik JM. Six degrees-of-freedom kinematics of ACL deficient knees during locomotion-compensatory mechanism. Gait Posture. 2003;17(1):34-42.

8. Yamaguchi S, Gamada K, Sasho T, Kato H, Sonoda M, Banks SA. In vivo kinematics of anterior cruciate ligament deficient knees during pivot and squat activities. Clin Biomech (Bristol, Avon). 2009;24(1):71-6.

9. Barrance PJ, Williams GN, Snyder-Mackler L, Buchanan TS. Altered knee kinematics in ACL-deficient non-copers: a comparison using dynamic MRI.J Orthop Res. 2006;24(2):132-40.

10. Ingersoll CD, Grindstaff TL, Pietrosimone BG, Hart JM. Neuromuscular consequences of anterior cruciate ligament injury. Clin Sports Med. 2008;27(3):383-404.

11. Phillips N, van Deursen RW. Landing stability in anterior cruciate ligament deficient versus healthy individuals: a motor control approach. Phys Ther Sport. 2008;9(4):193-201.

12. Andriacchi TP, Briant PL, Bevill SL, Koo S. Rotational changes at the knee after ACL injury cause cartilage thinning. Clin Orthop Relat Res. 2006;442:39-44.

13. Chaudhari AM, Briant PL, Bevill SL, Koo S, Andriacchi TP. Knee kinematics, cartilage morphology, and osteoarthritis after ACL injury. Med Sci Sports Exerc. 2008;40(2):215-22.

14. Farshad M, Gerber C, Meyer DC, Schwab A, Blank PR, Szucs T. Reconstruction versus conservative treatment after rupture of the anterior cruciate ligament: cost effectiveness analysis. BMC Health Serv Res. 2011;11:317.

15. Murray MM. Current status and potential of primary ACL repair. Clin Sports Med. 2009;28(1):51-61.

16. Kessler MA, Behrend H, Henz S, Stutz G, Rukavina A, Kuster MS. Function, osteoarthritis and activity after ACL-rupture: 11 years follow-up results of conservative versus reconstructive treatment. Knee Surg Sports Traumatol Arthrosc. 2008;16(5):442-8.

17. Cargalli E. Anterior cruciate ligament lesions: when and why to use knee braces. scribd inc; Available from: http://www.scribd. com/doc/52752709/Review-THE-ROLE-OF-KNEE-BRACES-AFTERACL-LESION.

18. Rebel M, Paessler HH. The effect of knee brace on coordination and neuronal leg muscle control: an early postoperative functional study in anterior cruciate ligament reconstructed patients. Knee Surg Sports Traumatol Arthrosc. 2001;9(5):272-81.

19. Cook FF, Tibone JE, Redfern FC. A dynamic analysis of a functional brace for anterior cruciate ligament insufficiency. Am J Sports Med.1989;17(4):519-24.

20. Seyed Mohseni S, Moss F, Karimi H, Kamali M. Arthrometric evaluation of stabilizing effect of knee functional bracing at different flexion angles.J Sports Sci Med. 2009;8(2):247-51.

21. Branch T, Hunter R, Reynolds P. Controlling anterior tibial displacement under static load: a comparison of two braces. Orthopedics. 1988;11(9):1249-52.

22. Hofmann AA, Wyatt RW, Bourne MH, Daniels AU. Knee stability in 
orthotic knee braces. Am J Sports Med. 1984;12(5):371-4

23. Wojtys EM, Loubert PV, Samson SY, Viviano DM. Use of a kneebrace for control of tibial translation and rotation. A comparison, in cadavera, of available models. J Bone Joint Surg Am. 1990;72(9):1323-9.

24. Campbell BM, Cipriani D, Yaggie JA. Influence of a Functional Knee Brace and Exercise on Lower Extremity Kinematics During Jogging. Clin Kinesiol . 2007;61(2):7.

25. De Vita P, Hortobagyi T. Functional knee brace alters predicted knee muscle and joint forces in people with ACL reconstruction during walking. J Appl Biomech . 2001;17(4):297-311.

26. Rahimi A, Wallace WA. Fuctional knee bracing for acl-deficient knees during gait.J Bone Joint Surg Br. 2003;85(suppI I):6-7.

27. Theoret D, Lamontagne M. Study on three-dimensional kinematics and electromyography of ACL deficient knee participants wearing a functional knee brace during running. Knee Surg Sports Traumatol Arthrosc. 2006;14(6):555-63.

28. Lucchetti L, Cappozzo A, Cappello A, Della Croce U. Skin movement artefact assessment and compensation in the estimation of knee-joint kinematics. J Biomech. 1998;31(11):977-84.

29. Akbarshahi M, Schache AG, Fernandez JW, Baker R, Banks S, Pandy MG. Non-invasive assessment of soft-tissue artifact and its effect on knee joint kinematics during functional activity. J Biomech. 2010;43(7):1292-301.

30. Ramsey DK, Lamontagne M, Wretenberg PF, Valentin A, Engstrom B, Nemeth G. Assessment of functional knee bracing: an in vivo three-dimensional kinematic analysis of the anterior cruciate deficient knee. Clin Biomech (Bristol, Avon). 2001;16(1):61-70.
31. Dejour H, Bonnin M. Tibial translation after anterior cruciate ligament rupture. Two radiological tests compared. J Bone Joint Surg Br.1994;76(5):745-9.

32. Scarvell JM, Smith PN, Refshauge KM, Galloway H, Woods K. Comparison of kinematics in the healthy and ACL injured knee using MRI.J Biomech. 2005;38(2):255-62.

33. Kozanek M, Hosseini A, de Velde SK, Moussa ME, Li JS, Gill TJ, et al. Kinematic evaluation of the step-up exercise in anterior cruciate ligament deficiency. Clin Biomech (Bristol, Avon). 2011;26(9):950-4.

34. Esfandiarpour F, Shakourirad A, Talebian Moghaddam S, Olyaei G, Eslami A, Farahmand F. Comparison of kinematics of ACL-deficient and healthy knees during passive flexion and isometric leg press. Knee. 2013;20(6):505-10.

35. Isaac DL, Beard DJ, Price AJ, Rees J, Murray DW, Dodd CA. In-vivo sagittal plane knee kinematics: ACL intact, deficient and reconstructed knees. Knee. 2005;12(1):25-31.

36. Vergis A, Hammarby S, Gillquist J. Fluoroscopic validation of electrogoniometrically measured femorotibial translation in healthy and ACL deficient subjects. Scand J Med Sci Sports. 2002;12(4):223-9.

37. Price AJ, Rees JL, Beard DJ, Gill RH, Dodd CA, Murray DM. Sagittal plane kinematics of a mobile-bearing unicompartmental knee arthroplasty at 10 years: a comparative in vivo fluoroscopic analysis. J Arthroplasty. 2004;19(5):590-7.

38. Marshall NW, Kotre CJ. Measurement and correction of the effects of lag on contrast-detail test results in fluoroscopy. Phys Med Biol. 2002;47(6):947-60. 